\title{
Metformin reduces risk of benign nodular goiter in patients with type 2 diabetes mellitus
}

\author{
Chin-Hsiao Tseng ${ }^{1,2,3}$ \\ ${ }^{1}$ Department of Internal Medicine, National Taiwan University College of Medicine, Taipei, Taiwan, ${ }^{2}$ Division of \\ Endocrinology and Metabolism, Department of Internal Medicine, National Taiwan University Hospital, Taipei, \\ Taiwan, and ${ }^{3}$ Division of Environmental Health and Occupational Medicine of the National Health Research \\ Institutes, Zhunan, Taiwan
}

Correspondence should be addressed to $\mathrm{C}-\mathrm{H}$ Tseng Email ccktsh@ms6.hinet.net

\begin{abstract}
Background: Whether metformin might affect the risk of benign nodular goiter in patients with type 2 diabetes mellitus has not been investigated.

Methods: Patients with new-onset type 2 diabetes mellitus during 1999-2005 were enrolled from Taiwan's National Health Insurance database. Analyses were conducted in a propensity score matched-pairs of 20,048 ever users and 20,048 never users of metformin. The patients were followed until December 31, 2011, for the incidence of benign nodular goiter. Hazard ratios were estimated by Cox regression incorporated with the inverse probability of treatment weighting using the propensity score.

Results: Among the never users and ever users of metformin, 392 and 221 cases were diagnosed of benign nodular goiter during follow-up, with incidence of 457.88 and 242.45 per 100,000 person-years, respectively. The overall hazard ratio for ever versus never users was 0.527 (95\% confidence interval: $0.447-0.621$ ). When cumulative duration of metformin therapy was divided into tertiles, the hazard ratios for the first ( $<25.3$ months), second ( $25.3-57.3$ months) and third (>57.3 months) tertiles were 0.815 (0.643-1.034), 0.648 (0.517-0.812) and $0.255(0.187-0.348)$, respectively. Sensitivity analyses estimating the overall hazard ratios for patients enrolled in each specific year from 1999 to 2005 consistently showed a lower risk of benign nodular goiter among users of metformin.

Conclusion: Metformin use is associated with a lower risk of benign nodular goiter in patients with type 2 diabetes mellitus.
\end{abstract}

\section{Introduction}

Insulin resistance plays an important role in the development of benign nodular goiter (1) and thyroid cancer $(2,3)$. Metformin is known for its insulin sensitizing effect resulting in the improvement of insulin resistance (4). In an early study in Taiwan, metformin use in patients with type 2 diabetes mellitus showed a protective effect on the development of thyroid cancer, with an adjusted hazard ratio of 0.683 (95\% confidence interval: 0.598-0.780) (5). Such a protective effect of metformin (c) 2019 European Society of Endocrinology Printed in Great Britain on thyroid cancer was also supported by a recent study conducted in Korea (6).

It is interesting that even though type 2 diabetes mellitus has been reported to increase the risk of several types of cancer, a study that used a random sample of the Taiwan's 2005 population showed that the risk of thyroid cancer was not increased in patients with type 2 diabetes mellitus in comparison to nondiabetes individuals (7). One of the possible explanations for such a lack of

Published by Bioscientifica Ltd. 
association between type 2 diabetes mellitus and thyroid cancer risk is because of the common use of metformin in the diabetes patients in Taiwan during the study period. Metformin has consistently stayed on the market in Taiwan for several decades together with sulfonylureas before other newer classes of oral antidiabetic drugs were available. Even during the period when biguanides were prohibited in the USA because of the potential risk of fatal lactic acidosis (8), metformin remained a mainstream of treatment for type 2 diabetes mellitus in Taiwan.

Thyroid cancer may develop from lesions diagnosed as benign nodular goiter and many patients with early thyroid cancer are diagnosed as having benign nodular goiter at the early stage (9). Therefore, it is important to investigate whether metformin may prevent the development of these early lesions. To the best of our knowledge, no previous epidemiological studies have ever investigated whether metformin might reduce the risk of benign nodular goiter in patients with type 2 diabetes mellitus, neither in the Asian nor in the non-Asian populations. The present population-based study aimed at investigating such a possible effect in a matched cohort of ever and never users of metformin enrolled from a nationwide dataset of the Taiwan's National Health Insurance (NHI).

\section{Subjects and methods}

The NHI is a universal healthcare system that covers more than $99 \%$ of the population in Taiwan. It has been implemented for more than 20 years since March 1995 and all in-hospitals and approximately $93 \%$ of all medical settings have contracts with the Bureau of the NHI. The reimbursement database of the NHI keeps all records of disease diagnoses, drug prescriptions and clinical procedures for each outpatient visit, use of emergency service and hospital admission of all insurants. The database, de-identified for the protection of privacy, can be used for academic research after ethics review and approval by the National Health Research Institutes. The present study was granted approval number 99274 . Informed consent was not required according to local regulations because the database has been de-identified for the protection of privacy.

During the study period, the International Classification of Diseases, Ninth Revision, Clinical Modification (ICD-9-CM) was used for disease diagnoses and diabetes was coded 250.XX. Benign nodular goiter was defined by ICD-9-CM codes 240 and 241. Patients with a diagnosis of thyroid cancer (193), hyperthyroidism
(242), congenital hypothyroidism (243), acquired hypothyroidism (244), thyroiditis (245) and other disorders of thyroid (246) were excluded.

The database has been described in detail elsewhere $(10,11)$. A 1:1 matched-pairs cohort of ever and never users of metformin was enrolled from the NHI database following the procedures shown in Fig. 1. At first, 423,949 patients who had a new-onset diabetes during 1999-2005 in the outpatient clinics and had received two or more prescriptions of antidiabetic drugs were identified. The following patients were then excluded: (1) ever users of metformin who had received other antidiabetic drugs before metformin was initiated $(n=183,837)$, (2) type 1 diabetes mellitus ( $n=2062)$, (3) missing data $(n=422)$, (4) diagnosis of thyroid disease at entry or within 6 months of diabetes diagnosis $(n=20,943)$, (5) patients with any cancer at entry or within 6 months of diabetes diagnosis $(n=23,317)$ and (6) follow-up $<180$ days $(n=14,510)$. As a result, 158,806 ever users and 20,052 never users of metformin were found (unmatched original cohort). Propensity score (PS) was created from all characteristics listed in Table 1 plus the date of entry by logistic regression. A matched-pairs cohort of 20,048 ever users and 20,048 never users (matched cohort) was then created by matching the PS based on the Greedy $8 \rightarrow 1$ digit match algorithm, as detailed elsewhere (12).

Potential confounders were divided into the following categories: (1) demographic data: age, sex, occupation and living region (classified as Taipei, Northern, Central, Southern, and Kao-Ping/Eastern); (2) major comorbidities: hypertension, dyslipidemia and obesity; (3) diabetesrelated complications: nephropathy, eye diseases, stroke, ischemic heart disease and peripheral arterial disease; (4) antidiabetic drugs: insulin, sulfonylureas, meglitinide, acarbose, rosiglitazone and pioglitazone; (5) commonly encountered comorbidities: chronic obstructive pulmonary disease (a surrogate for smoking), tobacco abuse, alcohol-related diagnoses, cancer, heart failure and ocular pterygium (a surrogate of UV sunlight exposure) and (6) commonly used medications in diabetes patients and drugs that potentially affect thyroid function: angiotensin-converting enzyme inhibitors/ angiotensin receptor blockers, calcium channel blockers, statins, fibrates, aspirin, amiodarone, lithium and use of contrast medium. Occupation was classified as class I (civil servants, teachers, employees of governmental or private businesses, professionals and technicians), class II (people without a specific employer, self-employed people or seamen), class III (farmers or fishermen) and class IV (low-income families supported by social welfare 
Newly diagnosed diabetes patients during 1999-2005 who had been followed up in the outpatient clinics with prescription of antidiabetic drugs for 2 or more times

$\mathrm{N}=423,949$

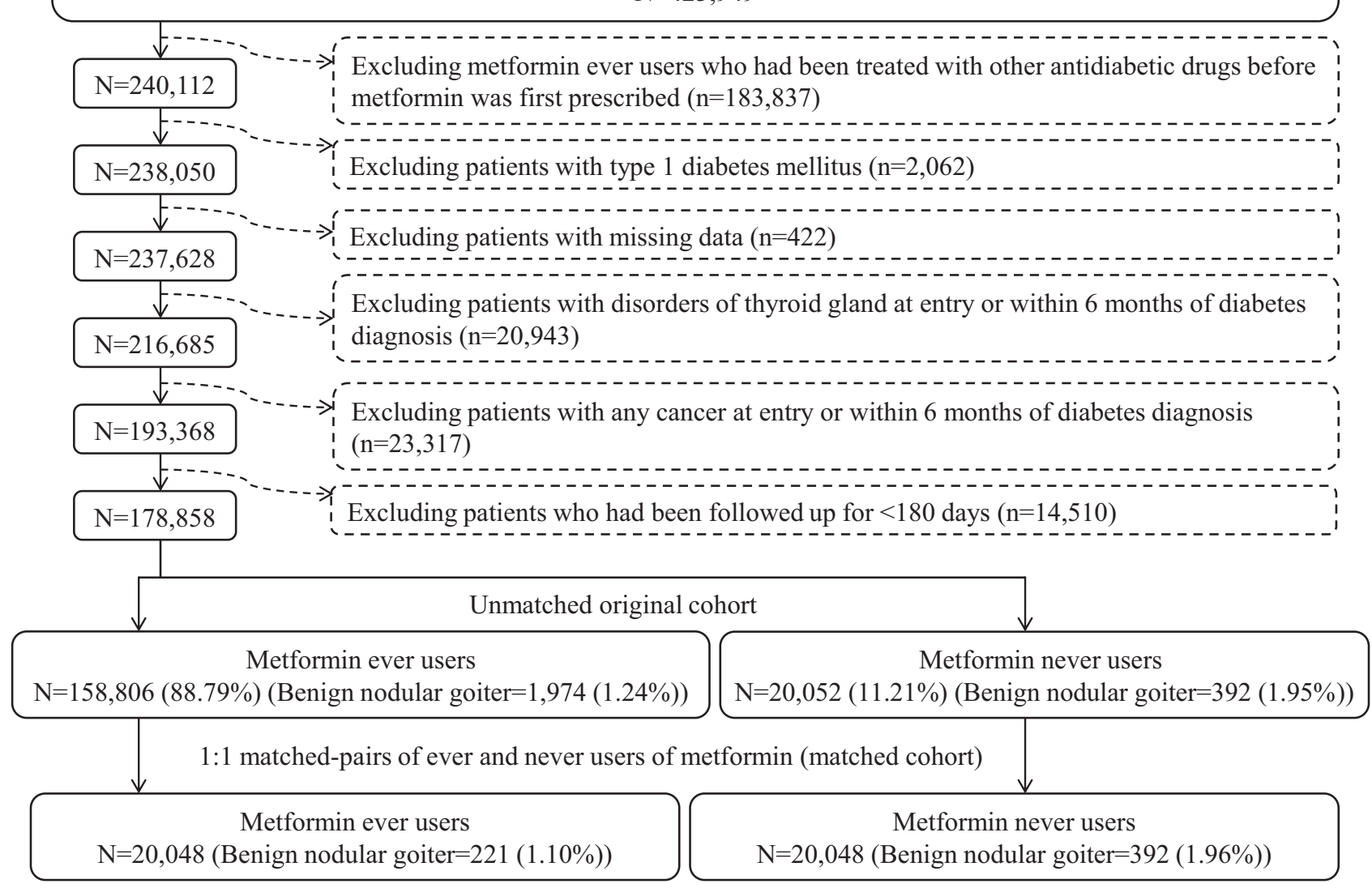

\section{Figure 1}

Flowchart showing the procedures in creating a cohort of 1:1 matched-pairs of metformin ever and never users from the reimbursement database of the National Health Insurance.

or veterans). The ICD-9-CM codes for the above diagnoses were: hypertension (401-405), dyslipidemia (272.0-272.4), obesity (278), nephropathy (580-589), eye diseases (250.5: diabetes with ophthalmic manifestations, 362.0: diabetic retinopathy, 369: blindness and low vision, 366.41: diabetic cataract, and 365.44: glaucoma associated with systemic syndromes), stroke (430-438), ischemic heart disease (410-414), peripheral arterial disease (250.7, 785.4, 443.81 and 440-448), chronic obstructive pulmonary disease (490-496), tobacco abuse (305.1, 649.0 and 989.84), alcohol-related diagnoses (291, 303, 535.3, 571.0571.3 and 980.0), cancer (140-208), heart failure (398.91, 402.11, 402.91, 404.11, 404.13, 404.91, 404.93 and 428) and ocular pterygium (372.40-372.44).

Standardized difference was calculated for each covariate as a test of balance diagnostic. Austin and Stuart recommended the use of a cutoff value $>10 \%$ as an indicator of potential confounding from the variable (13).
Cumulative duration of metformin therapy in months was calculated from the database. Its tertiles were used to evaluate the possibility of a dose-response relationship. Incidence density of benign nodular goiter was calculated for never users, ever users and the tertiles of cumulative duration of metformin therapy. The numerator of the incidence was the case number of new cases of benign nodular goiter diagnosed during follow-up. The denominator expressed as person-years was the follow-up duration, which ended on December 31, 2011, at the time of a new diagnosis of benign nodular goiter or on the date of death or the last reimbursement record.

Cox proportional hazards regression model incorporated with the inverse probability of treatment weighting (IPTW) using the PS was used to estimate hazard ratios and their 95\% confidence intervals for ever users and for each tertile of cumulative duration in comparison to never users. This method was proposed by Austin to 
Table 1 Characteristics in never and ever users of metformin. Data are presented as $n$ (\%).

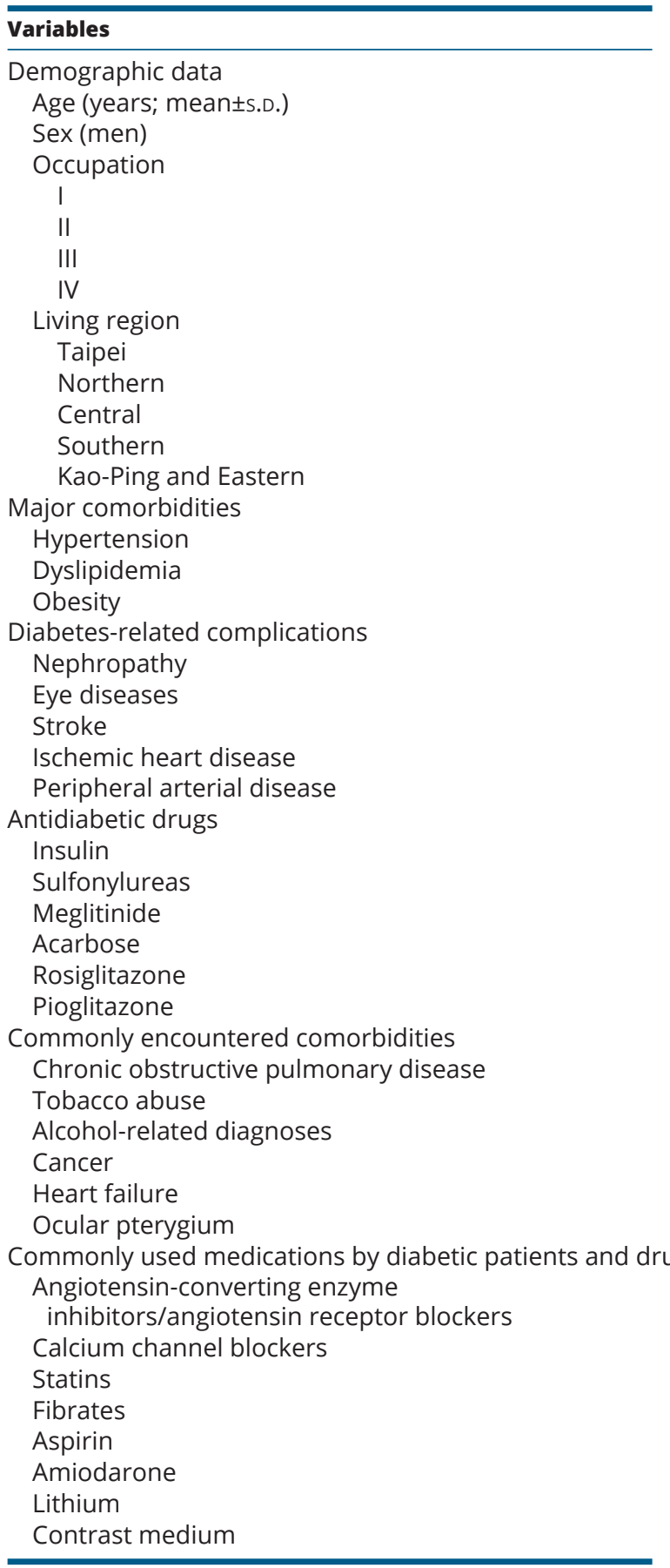

Never users, $(n=20048)$

$68.82 \pm 13.27$

11348 (56.60)

7171 (35.77)

3375 (16.83)

4865 (24.27)

4637 (23.13)

6677 (33.31)

2147 (10.71)

3454 (17.23)

3548 (17.70)

4222 (21.06)

17072 (85.16)

13895 (69.31)

439 (2.19)

7711 (38.46)

3462 (17.27)

8139 (40.60)

10663 (53.19)

5165 (25.76)

1751 (8.73)

14480 (72.23)

1784 (8.90)

$2146(10.70)$

$561(2.80)$

444 (2.21)

11023 (54.98)

$480(2.39)$

$1314(6.55)$

2069 (10.32)

$5758(28.72)$

1152 (5.75)

that potentially

14767 (73.66)

13623 (67.95)

10371 (51.73)

6534 (32.59)

12512 (62.41)

1191 (5.94)

$85(0.42)$

$8560(42.70)$
Ever users, $(n=20048)$

$68.62 \pm 12.19$

$-0.99$

11334 (56.53)

$-0.19$

7296 (36.39)

$3485(17.38) \quad 1.34$

$4770(23.79) \quad-0.96$

$4497(22.43) \quad-1.59$

6721 (33.52)

$2121(10.58) \quad-0.44$

$3358(16.75) \quad-1.27$

$3601(17.96) \quad 0.84$

$4247(21.18) \quad 0.44$

$17039(84.99) \quad-0.36$

$13994(69.80) \quad 1.09$

$441(2.20)-0.03$

$7530(37.56) \quad-2.16$

$3322(16.57) \quad-2.30$

$8163(40.72) \quad 0.38$

$10691(53.33) \quad 0.38$

$5027(25.07) \quad-1.69$

$1572(7.84) \quad-5.11$

$14982(74.73)$

$1768(8.82) \quad-0.65$

$2272(11.33) \quad 0.32$

$579(2.89) \quad-0.17$

$458(2.28) \quad-0.33$

$10907(54.40) \quad-1.02$

$514(2.56) \quad 1.08$

$1283(6.40) \quad-0.77$

$2067(10.31) \quad-0.06$

$5646(28.16) \quad-1.36$

$1146(5.72) \quad-0.01$

0.12

0.51

$-0.22$

$-0.32$

$-0.43$

$-0.10$

$-0.09$

0.24

Refer to 'Subjects and methods' section for the classification of occupation.

reduce the potential confounding from the differences in characteristics between two compared groups (14).

In consideration that more antidiabetic drugs have been introduced into the market of Taiwan after 1995 and the guidelines for the use of antidiabetic drugs have evolved over the last two decades, the PS-weighted hazard ratios for benign nodular goiter for ever versus never users of metformin were also estimated for each specific year from 1999 to 2005.

Analyses were conducted using SAS statistical software, version 9.3 (SAS Institute). $P<0.05$ was considered statistically significant. 


\section{Results}

Table 1 shows the characteristics in never and ever users of metformin. None of the variables had a value of standardized difference $>10 \%$, suggesting that the two groups were well matched in potential confounders.

Table 2 shows the incidence of benign nodular goiter and the hazard ratios by metformin exposure. The overall hazard ratios indicated a significantly lower risk in metformin users and the tertile analyses suggested a doseresponse relationship. Metformin use for 2 or more years in the second and third tertiles showed a significantly reduced risk, while those in the first tertile showed a lower risk with borderline significance.

Table 3 shows the overall hazard ratios for patients enrolled in each specific year from 1999 to 2005. The beneficial effect of metformin could be consistently observed in patients enrolled in different years. It was also observed that there was a gradual increase of proportions of never users from $37.73 \%$ in 1999 to $65.37 \%$ in 2005 .

\section{Discussion}

The is the first population-based observational study that showed a reduced risk of benign nodular goiter associated with metformin use in patients with type 2 diabetes mellitus in a dose-response pattern (Table 2). The reduced risk associated with metformin use was especially significant when it had been used for 2 or more years (Table 2). Such a beneficial effect was consistent disregarding the year in which the patients were enrolled (Table 3). The findings of the present study together with our previous observation that metformin use is associated with a lower risk of thyroid cancer (5) imply that metformin may prevent the diverted growth of thyrocytes from benign nodular goiter to thyroid cancer.
The mechanisms of a reduced risk of benign nodular goiter associated with metformin use requires more investigation, but some biological actions of metformin could explain such a beneficial effect. Insulin resistance is associated with an impairment in thyroid function and may affect the morphological changes of the thyroid gland (15). Metformin inhibits the mitochondrial respiratory-chain complex 1 , leading to an activation of the liver kinase B1 (LKB1)/5'-adenosine monophosphateactivated protein kinase (AMPK) pathway, which in turn inhibits gluconeogenesis in the liver, lowers blood glucose and improves insulin resistance (16). Metformin may also improve insulin resistance by stimulating the expression of insulin receptor and activation of tyrosine kinase (17). Furthermore, metformin may change the composition of gut microbiota with an increase in Akkermansia species, which is responsible for the improvement in insulin resistance and reduction in tissue inflammation (18). Thyrocyte proliferation is stimulated by thyroid-stimulating hormone via activation of the mammalian target of rapamycin (mTOR) pathways (19) and metformin is well known for its inhibitory effect on mTOR via activation of LKB1/AMPK (20). In animals, metformin suppressed thyroid-stimulating hormone and increased free triiodothyronine and free thyroxine (21). In obese patients with insulin resistance and euthyroid goiter, treatment with both metformin and levothyroxine significantly lowered thyroid-stimulating hormone and improved metabolic parameters (including waist/hip ratio, visceral fat thickness, insulin level and insulin resistance) better than treatment with levothyroxine alone (22). Two recent meta-analyses do show that metformin is effective in reducing the size of benign thyroid nodules together with a reduction of thyroid-stimulating hormone in humans $(23,24)$.

It is worthy to note that the proportions of ever users of metformin decreased gradually from 1999 to 2005 (Table 3). This could be because of the more and

Table 2 Incidence of benign nodular goiter and hazard ratios by metformin exposure.

\begin{tabular}{|c|c|c|c|c|c|c|c|}
\hline Metformin use & $n$ & Total n & Person-year & $\begin{array}{c}\text { Incidence rate } \\
\text { (per } 100000 \text { person-years) }\end{array}$ & $\begin{array}{l}\text { Hazard } \\
\text { ratio }\end{array}$ & $\begin{array}{l}\text { 95\% Confidence } \\
\text { interval }\end{array}$ & $P$ \\
\hline Never users & 392 & 20048 & 85611.55 & 457.88 & 1.000 & & \\
\hline Ever users & 221 & 20048 & 91153.76 & 242.45 & 0.527 & $(0.447-0.621)$ & $<0.01$ \\
\hline \multicolumn{8}{|c|}{ Tertiles of cumulative duration of metformin therapy (months) } \\
\hline Never users & 392 & 20048 & 85611.55 & 457.88 & 1.000 & & \\
\hline$<25.3$ & 83 & 6612 & 21863.57 & 379.63 & 0.815 & $(0.643-1.034)$ & 0.09 \\
\hline $25.3-57.3$ & 93 & 6626 & 30996.89 & 300.03 & 0.648 & $(0.517-0.812)$ & $<0.01$ \\
\hline$>57.3$ & 45 & 6810 & 38293.30 & 117.51 & 0.255 & $(0.187-0.348)$ & $<0.01$ \\
\hline
\end{tabular}


Table 3 Hazard ratios for benign nodular goiter for ever versus never users of metformin estimated for each specific year from 1999 to 2005.

\begin{tabular}{|c|c|c|}
\hline \multirow[b]{2}{*}{ Year } & \multicolumn{2}{|c|}{ Ever users } \\
\hline & Cases followed & $\%$ \\
\hline 1999 & 3096 & 62.27 \\
\hline 2000 & 3105 & 61.24 \\
\hline 2001 & 3006 & 56.32 \\
\hline 2002 & 2948 & 52.24 \\
\hline 2003 & 2898 & 48.40 \\
\hline 2004 & 2566 & 42.25 \\
\hline 2005 & 2429 & 34.63 \\
\hline
\end{tabular}

\begin{tabular}{c}
\hline \multicolumn{2}{c}{ Never users } \\
\hline Cases followed \\
\hline 1876 \\
1965 \\
2331 \\
2695 \\
3089 \\
3507 \\
4585 \\
\hline
\end{tabular}

more antidiabetic drugs being introduced into Taiwan after 1995. However, the protective effect of metformin remained consistent in each specific year of patients' enrollment (Table 3). Because metformin is protective for different types of cancer including thyroid cancer (5) and benign nodular goiter in the present study (Tables 2 and 3), the decreasing rates of metformin use might potentially increase the burden of cancer in the diabetes patients if more newer classes of oral antidiabetic drugs are introduced into clinical practice and metformin is no longer prescribed to the patients. It is also worthy to note that the beneficial effect of metformin may only be significant after a prolonged use of probably more than 2 years (Table 2). At this moment, in the absence of contraindications, it is reasonable to recommend the persistent use of metformin in patients with type 2 diabetes mellitus even when the addition of other antidiabetic drugs is required for glycemic control. In consideration that metformin is effective in reducing the size of benign thyroid nodules and reducing thyroidstimulating hormone levels in humans $(23,24)$ and that it is cheap and safe and would not cause hypoglycemia when used as monotherapy, it may be a good choice for patients with type 2 diabetes mellitus and an existing benign nodular goiter.

The present study has been conducted with careful consideration of common methodological limitations seen in many earlier pharmaco-epidemiological studies. These may include selection bias, prevalent user bias, immortal time bias and confounding by indication.

Selection bias can be avoided by using the nationwide database of the NHI that covers more than $99 \%$ of the population. By enrolling patients with new-onset diabetes and new users of metformin, prevalent user bias can be prevented. Because metformin ever users were enrolled from those who received metformin as the first antidiabetic drug (Fig. 1), the potential confounding effect of other antidiabetic drugs used before metformin was initiated could also be avoided.

\begin{tabular}{c}
\hline Hazard ratio \\
\hline 0.600 \\
0.419 \\
0.465 \\
0.502 \\
0.438 \\
0.613 \\
0.459 \\
\hline
\end{tabular}

\begin{tabular}{cccc}
\hline 95\% Confidence interval & & P value \\
\cline { 1 - 1 }$(0.385-0.935)$ & & $<0.05$ \\
$(0.274-0.640)$ & & $<0.01$ \\
$(0.302-0.719)$ & & $<0.01$ \\
$(0.329-0.766)$ & & $<0.01$ \\
$(0.274-0.700)$ & & $<0.01$ \\
$(0.395-0.951)$ & & $<0.05$ \\
$(0.274-0.767)$ & & $<0.01$ \\
\hline
\end{tabular}

Bias from immortal time, the follow-up period during which the outcome cannot happen, can be introduced when the treatment status and/or the follow-up time are inappropriately assigned (25). In the present study, because only patients who had been prescribed antidiabetic drugs for two or more times were enrolled (Fig. 1), most cases with indefinite diagnosis of diabetes mellitus should have been excluded. Because the NHI is a universal healthcare system with very high coverage rate and the information of prescriptions was complete during the long follow-up period, it was unlikely that the treatment status would be misclassified. The immortal time within the period from the diagnosis of diabetes mellitus to the start of prescription of antidiabetic drugs or during the period incurred in patients who had been followed for a short period of time (<180 days) was not included in the calculation of person-years in the present study. Because all discharge drugs can be obtained directly from the hospital when patients are discharged in Taiwan, the immortal time during the waiting period from drug prescription to drug dispense after discharge would not happen here.

Although confounding by indication might have been reduced by the enrollment of a well-matched cohort (Table 1) and the use of the Cox proportional hazards regression model incorporated with IPTW (Table 2), we could not exclude residual confounding from data incompleteness and misclassification. Furthermore, unmeasured confounders remain an issue. The consistency of the findings in patients enrolled during different years (Table 3) suggested that the beneficial effect of metformin on benign nodular goiter would not be affected by the changes in the recommendations of the use of antidiabetic drugs that evolved during the long period of time.

The present study has some additional merits. The use of medical records should have significantly reduced the bias related to self-reporting. Detection bias related to different socioeconomic status can be a problem in some countries. However, this was less likely in Taiwan because 
the drug cost-sharing in the NHI system is low and much expenses can be waived in patients with low-income, in veterans or when the patients receive prescription refills for chronic disease.

There are some limitations in the study that require mentioning. First, we did not have measurement data of confounders like anthropometric factors, cigarette smoking, alcohol drinking, lifestyle, physical activity, nutritional status, dietary pattern, family history, genetic parameters, education level, iodine intake and selenium status (26). Second, it remained unknown what criteria were used by the attending doctors to add the diagnosis of benign nodular goiter. Third, the lack of biochemical data, thyroid hormones, glucose and insulin levels and indicators of beta cell function and insulin resistance did not allow additional in-depth investigation of some potential mechanisms. Fourth, because the growth rate of benign nodular goiter is usually very slow, the biological plausibility of the beneficial effect of metformin to be significant at 2 years (Table 2) requires further investigation. Fifth, because only a small proportion (7-15\%) of patients with benign nodular goiter will develop thyroid cancer (most of them are well differentiated) (27), whether the beneficial effects of metformin on benign nodular goiter as shown in the present study (Table 2) may contribute to a reduced risk of thyroid cancer associated with its use $(5,6)$ requires additional investigation. Therefore, high-quality clinical trials or prospective observational studies with large sample size are needed to confirm the beneficial effect of metformin on benign nodular goiter. At this moment it is too soon to recommend the use of metformin as a preventive or therapeutic agent for thyroid disease.

In summary, this population-based retrospective cohort study reports a reduced risk of benign nodular goiter associated with metformin use in patients with type 2 diabetes mellitus. However, confirmation with prospective cohort study designs or clinical trials is necessary. Furthermore, its beneficial effects in nondiabetes people are worthy of further investigation.

\section{Declaration of interest}

The author declares that there is no conflict of interest that could be perceived as prejudicing the impartiality of this study.

\section{Funding}

The study was supported by the Ministry of Science and Technology (MOST 107-2221-E-002-129-MY3) of Taiwan. The funders had no role in study design, data collection and analysis, decision to publish or preparation of the manuscript.
Author contribution statement

Tseng $\mathrm{C} \mathrm{H}$ researched data and wrote manuscript.

\section{Acknowledgments}

The study is based in part on data from the National Health Insurance Research Database provided by the Bureau of National Health Insurance, Department of Health and managed by National Health Research Institutes. The interpretation and conclusions contained herein do not represent those of Bureau of National Health Insurance, Department of Health or National Health Research Institutes. The guarantor of this manuscript is Tseng $\mathrm{CH}$.

\section{References}

1 Tsatsoulis A. The role of insulin resistance/hyperinsulinism on the rising trend of thyroid and adrenal nodular disease in the current environment. Journal of Clinical Medicine 20187 E37. (https://doi. org/10.3390/jcm7030037)

2 Malaguarnera R, Vella V, Nicolosi ML \& Belfiore A. Insulin resistance: any role in the changing epidemiology of thyroid cancer? Frontiers in Endocrinology 20178 314. (https://doi.org/10.3389/ fendo.2017.00314)

3 Heidari Z, Abdani M \& Mansournia MA. Insulin resistance associated with differentiated thyroid carcinoma: penalized conditional logistic regression analysis of a matched case-control study data. International Journal of Endocrinology and Metabolism 201816 e14545. (https://doi. org/10.5812/ijem.14545)

4 Wang YW, He SJ, Feng X, Cheng J, Luo YT, Tian L \& Huang Q. Metformin: a review of its potential indications. Drug Design, Development and Therapy 201711 2421-2429. (https://doi. org/10.2147/DDDT.S141675)

5 Tseng $\mathrm{CH}$. Metformin reduces thyroid cancer risk in Taiwanese patients with type 2 diabetes. PLoS ONE 20149 e109852. (https://doi. org/10.1371/journal.pone.0109852)

6 Cho YY, Kang MJ, Kim SK, Jung JH, Hahm JR, Kim TH, Nam JY, Lee BW, Lee YH, Chung JH et al. Protective effect of metformin against thyroid cancer development: a population-based study in Korea. Thyroid 201828 864-870. (https://doi.org/10.1089/ thy.2017.0550)

7 Tseng $\mathrm{CH}$. Thyroid cancer risk is not increased in diabetic patients. PLoS ONE 20127 e53096. (https://doi.org/10.1371/journal. pone.0053096)

8 Bailey CJ. Metformin: historical overview. Diabetologia 201760 1566-1576. (https://doi.org/10.1007/s00125-017-4318-z)

9 Glynn N, Hannon MJ, Lewis S, Hillery P, Al-Mousa M, Hill AD, Keeling F, Morrin M, Thompson CJ, Smith D et al. Utility of repeat cytological assessment of thyroid nodules initially classified as benign: clinical insights from multidisciplinary care in an Irish tertiary referral centre. BMC Endocrine Disorders 201616 45. (https:// doi.org/10.1186/s12902-016-0125-7)

10 Tseng CH. Metformin is associated with a lower risk of colorectal cancer in Taiwanese patients with type 2 diabetes: a retrospective cohort analysis. Diabetes and Metabolism 201743 438-445. (https:// doi.org/10.1016/j.diabet.2017.03.004)

11 Tseng $\mathrm{CH}$. Metformin and lung cancer risk in patients with type 2 diabetes mellitus. Oncotarget 20178 41132-41142. (https://doi. org/10.18632/oncotarget.17066)

12 Parsons LS. Performing a 1:N case-control match on propensity score. (available at: http://www.google.com.tw/url?sa=t\&rct=j\&q= \&esrc $=$ s\&source $=$ web $\& \mathrm{~cd}=1 \&$ ved $=0 \mathrm{CBsQFjAAahUKEwibi7HllcnIAh}$ UDoJQKHVeZA9A\&url=http\%3A\%2F\%2Fwww2.sas.com\%2Fproce edings\%2Fsugi29\%2F165-29.pdf\&usg=AFQjCNFOHGWYu8E8Bn4Bo1TUiJKtT987Q). Accessed on 19 March 2019. 
13 Austin PC \& Stuart EA. Moving towards best practice when using inverse probability of treatment weighting (IPTW) using the propensity score to estimate causal treatment effects in observational studies. Statistics in Medicine 201534 3661-3679. (https://doi. org/10.1002/sim.6607)

14 Austin PC. The performance of different propensity score methods for estimating marginal hazard ratios. Statistics in Medicine 201332 2837-2849. (https://doi.org/10.1002/sim.5705)

15 Meng X, Xu S, Chen G, Derwahl M \& Liu C. Metformin and thyroid disease. Journal of Endocrinology 2017233 R43-R51. (https://doi. org/10.1530/JOE-16-0450)

16 Rena G, Pearson ER \& Sakamoto K. Molecular mechanism of action of metformin: old or new insights? Diabetologia 201356 1898-1906. (https://doi.org/10.1007/s00125-013-2991-0)

17 Viollet B, Guigas B, Sanz Garcia N, Leclerc J, Foretz M \& Andreelli F. Cellular and molecular mechanisms of metformin: an overview. Clinical Science 2012122 253-270. (https://doi.org/10.1042/CS20110386)

18 Hur KY \& Lee MS. New mechanisms of metformin action: focusing on mitochondria and the gut. Journal of Diabetes Investigation 20156 600-609. (https://doi.org/10.1111/jdi.12328)

19 Morshed SA, Ma R, Latif R \& Davies TF. How one TSH receptor antibody induces thyrocyte proliferation while another induces apoptosis. Journal of Autoimmunity 201347 17-24. (https://doi. org/10.1016/j.jaut.2013.07.009)

20 Mallik R \& Chowdhury TA. Metformin in cancer. Diabetes Research and Clinical Practice 2018143 409-419. (https://doi.org/10.1016/j. diabres.2018.05.023)
21 Hu X, Liu Y, Wang C, Hou L, Zheng X, Xu Y, Ding L \& Pang S. Metformin affects thyroid function in male rats. Oncotarget $2017 \mathbf{8}$ 107589-107595. (https://doi.org/10.18632/oncotarget.22536)

22 Răcătăianu N, Bolboacă SD, Sitar-Tăut AV, Mârza S, Moga D, Valea A \& Ghervan C. The effect of metformin treatment in obese insulin-resistant patients with euthyroid goiter. Acta Clinica Belgica 201873 317-323. (https://doi.org/10.1080/17843286.201 8.1439273)

23 Sui M, Yu Y, Zhang H, Di H, Liu C \& Fan Y. Efficacy of metformin for benign thyroid nodules in subjects with insulin resistance: a systematic review and meta-analysis. Frontiers in Endocrinology 20189 494. (https://doi.org/10.3389/fendo.2018.00494)

24 He X, Wu D, Hu C, Xu T, Liu Y, Liu C, Xu B \& Tang W. Role of metformin in the treatment of patients with thyroid nodules and insulin resistance: a systematic review and meta-analysis. Thyroid 201929 359-367. (https://doi.org/10.1089/thy.2017.0707)

25 Lévesque LE, Hanley JA, Kezouh A \& Suissa S. Problem of immortal time bias in cohort studies: example using statins for preventing progression of diabetes. BMJ $2010 \mathbf{3 4 0}$ b5087. (https://doi. org/10.1136/bmj.b5087)

26 Knudsen N \& Brix TH. Genetic and non-iodine-related factors in the aetiology of nodular goitre. Best Practice and Research: Clinical Endocrinology and Metabolism 201428 495-506. (https://doi. org/10.1016/j.beem.2014.02.005)

27 Cohen RN \& Davis AM. Management of adult patients with thyroid nodules and differentiated thyroid cancer. JAMA $2017 \mathbf{3 1 7} 434-435$. (https://doi.org/10.1001/jama.2016.18420)

Received 27 February 2019

Revised version received 27 March 2019

Accepted 15 April 2019 